\title{
Magnetic field strength properties in bone marrow during pulsed electromagnetic stimulation
}

\author{
Hiroyuki Tamaki ${ }^{1,2}$, Kengo Yotani ${ }^{3}$, Atsumu Yuki ${ }^{4}$, Hikari Kirimoto ${ }^{1}$, Kazuhiro Sugawara ${ }^{1,2}$, \\ Hideaki Onishi ${ }^{1,2}$
}

\footnotetext{
${ }^{1}$ Institute for human movement and Medical Sciences, Japan;

${ }^{2}$ Department of Physical Therapy, Niigata University of Health and Welfare, Niigata, Japan;

${ }^{3}$ Department of Physiological Sciences, National Institute of Fitness and Sports, Kanoya, Japan;

${ }^{4}$ Department of Physical Education, Aichi University of Education, Kariya, Japan.

Email: hiroyuki-tamaki@nuhw.ac.jp
}

Received 4 October 2010; revised 7 October 2010; accepted 8 October 2010.

\begin{abstract}
We clarified the characteristics of pulsed electromagnetic field (PEMF) strength in marrow cavity with bone marrow in long bones based on actual measurements taken during pulsed magnetic stimulation (PMS). Measurements were made under 810 different conditions of stimulation intensity, distance, and position. Significant and strong linear correlations were observed between PEMF strength and stimulation intensity. PEMF strength in marrow cavity during PMS showed an exponential decay depending on coil-sensor distance, with a breaking point at approximately $30 \mathrm{~mm}$. PEMF strength distributions in bone showed geometric differences between 3 types. These findings suggest that PEMF strength in bone depends on stimulation intensity, distance and horizontal position. Our actual measured data could be useful in determining stimulation programs and estimating the in vivo efficacy of PEMF in marrow cavity for research and clinical use.
\end{abstract}

Keywords: Magnetic Stimulation; Bone Tissue; Focality; Geometry; Intensity

\section{INTRODUCTION}

Pulsed electromagnetic field (PEMF) stimulation has been used clinically to treat bone disorders and reportedly promotes osteogenesis, in part through direct actions on osteoblasts. However, such clinical success contrasts with negative reports regarding the effects of pulsed magnetic stimulation on cellular proliferation, differentiation and mineralization of osteoblasts in vitro $[1,2]$. Differences in response to biophysical factors seemed to depend on the specific conditions of pulsed magnetic stimulation, e.g., stimulation intensity, fre- quency, exposure time and stage of osteoblast maturation [3]. In particular, stimulation intensity depends on stimulator output, distance and position [4,5]. Only a few in vivo studies have shown PEMF effects on bone formation processes in tissue, protein and mRNA levels [6-8]. An accompanying problem is the difficulty in determining magnetic field strength inside bone in vivo, as characterization of magnetic field strength in bone during PEMF stimulation under different conditions remains incomplete. A measurement of PEMF strength in marrow cavity into which a magnetic sensor probe has been inserted would enable us to obtain the data applicable to in vivo studies [5,8]. This study clarified the characteristics of PEMF strength in marrow cavity with bone marrow in long bones during pulsed magnetic stimulation under 810 different conditions of stimulation intensity, distance, and positions.

\section{METHODS}

The end of a magnetic sensor probe (length, $101.6 \mathrm{~mm}$; sensor location, $8.5 \mathrm{~mm}$ from bone end; active area, 3.8-mm diameter) was placed into the distal metaphysis of a femur removed from male Wistar rats at 30-33 weeks old $(n=6)$. Three-dimensional micro-CT images of long bones (Skyscan 1076®, Skyscan, Aartselaar, Belgium) were obtained to confirm the positions of sensor and wire in the marrow cavity (Figure 1) [8].

Bones were set on a precision horizontal stage that was able to be manually moved in 3 dimensions ( $\mathrm{X}, \mathrm{Y}$ and $\mathrm{Z}$ axes). A 9-cm-diameter magnetic coil was held in a clamp horizontally and placed with the center of the coil right above the active area of the sensor probe. The magnetic coil and sensor probe were connected to a magnetic stimulator (Magstim 200; The Magstim Company, Whitland, UK) and a gaussmeter (5180; FW Bell, Orland, USA), respectively. The PEMF was generated 


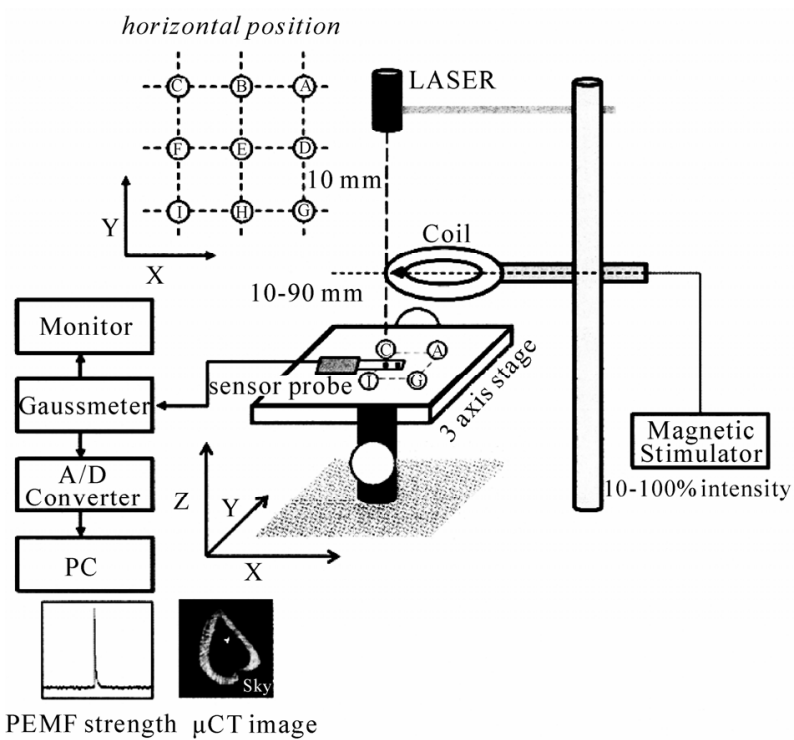

Figure 1. A schematic of the experimental setup. Measurements were performed at 9 horizontal positions (A-I) on the precision stage (X-Y axis) and at 9 coil-sensor distances. Sensor position was confirmed with $\mu \mathrm{CT}$ scanner and a laser beam so as to be centred $(\mathrm{E})$ on the $3 \times 3$ grid points at 10 -mm intervals before the experiment.

by a commercially available, clinically approved magnetic stimulator (Magstim 200; The Magstim Company) which was able to generate a maximum magnetic field of $2 \mathrm{~T}$. Stimuli were delivered at 10 stimulation intensities from $10-100 \%$ in increments of $10 \%$ of the maximal stimulator output. A total of 81 different measurement positions were set to determine a distribution map of individual magnetic field strengths in marrow cavity using the 3-dimensional drive precision stage, that is, at 9 coil-to-sensor distances from 10-90 $\mathrm{mm}$ with intervals of $10 \mathrm{~mm}$ ( $\mathrm{Z}$ axis) and at 9 horizontal positions on $3 \times 3$ grid points with intervals of $10 \mathrm{~mm}(\mathrm{X}-\mathrm{Y}$ axes) (Figure 1). Peak PEMF strength (magnetic flux density) during PEMF stimulation was measured 3 times for each condition using the gaussmeter (5180; FW Bell) [9] with an analog output of $100 \mathrm{kHz}$ sampling rate, $\pm 0.75 \%$ accuracy and DC-30 kHz bandwidth. Signals from each measurement were checked using an oscilloscope and subsequently digitized at a sampling frequency of 100 $\mathrm{kHz}$ using a 16-bit $\mathrm{A} / \mathrm{D}$ converter (Power Lab; AD Instruments, Tokyo, Japan) and stored on a personal computer for later analysis. Each instrument was calibrated immediately before data collection. The same procedure for measurement was followed without the bone at the end of a magnetic sensor probe as a control. Repeatability was assessed on 2 separate days in a pilot study under conditions of $30 \mathrm{~mm}$ magnetic sensor-coil distance and position $\mathrm{E}$ as in Figure 1 on the $\mathrm{X}-\mathrm{Y}$ axis at stimulation intensities of $10-100 \%$.
Precision of measurements for peak PEMF strength was calculated as the percent coefficient of variation (CV\%) using data from 100 repeated measurements under each individual condition. Bland-Altman analysis [10] was used to evaluate systemic bias and the limits of agreement between measured values obtained on test-retest. Linear regression analysis was used to determine correlations between stimulation intensity and PEMF strength values. Non-linear equations were used to describe the relationship between PEMF strength and sensor-coil distance. Analysis of variance (ANOVA) was used to compare PEMF strength as a function of stimulation intensity, sensor-coil distance and position. Data are presented as mean \pm standard deviation (SD), with values of $\mathrm{P}<0.05$ considered statistically significant. All study protocols were approved by the ethics committee of the National Institute of Fitness and Sports and conducted in compliance with the Declaration of Helsinki.

\section{RESULTS}

$\mathrm{CV} \%$ for repeated measurements and Pearson's correlation coefficient for the test-retest of PEMF strength measurements in bone during PEMF stimulation were 0.19$0.97 \%$ and $r=1.00(\mathrm{P}<0.0001)$, respectively. BlandAltman analysis showed a mean difference (bias) of 0.1 $\mathrm{mT}$ and $95 \%$ limits of agreement of $\pm 1.21 \mathrm{mT}$, demonstrating excellent agreement between test-retest values.

A significant strong linear correlation was seen between PEMF strength and stimulation intensity at each position and coil-sensor distance $\left(\mathrm{r}^{2}=0.99-1.00, \mathrm{P}<\right.$ 0.0001) (Figure 2). PEMF strength in marrow cavity during PEMF stimulation showed an exponential decay depending on coil-sensor distance at each stimulation intensity, with a breaking point at approximately $30 \mathrm{~mm}$ distance, except for at positions $\mathrm{C}, \mathrm{F}$ and $\mathrm{I}\left(\mathrm{r}^{2}=\right.$ 0.97-0.99, $\mathrm{P}<0.0001$ ) (Figure 3). Significant reverse correlations were noted between PEMF strength and coil-sensor distance in positions $\mathrm{C}, \mathrm{F}$ and I under all stimulation intensity conditions $\left(\mathrm{r}^{2}=0.84-0.96, \mathrm{P}<\right.$ 0.0001 ). Horizontal positions were classified into 3 groups exhibiting the same trends of regression lines and curves according to horizontal position, with the highest values for positions A-D-E, middle values for B-G-H, and lowest values for C-F-I. For instance, mean PEMF strength for each position at $50 \%$ stimulation intensity from $30 \mathrm{~mm}$ distance were $163.7 \pm 4.8 \mathrm{mT}, 128.3 \pm 2.1$ $\mathrm{mT}$ and $90.0 \pm 2.9 \mathrm{mT}$ for high, middle and low values groups, respectively (Figure 4). Intra-group comparison of each regression slope did not reveal any significant differences $(\mathrm{P}>0.36)$ among regression lines for positions A, D and E, and likewise for the B-G-H and C-F-I groups. For inter-group comparison between position A-D-E, B-G-H and C-F-I groups, significant differences 
Control
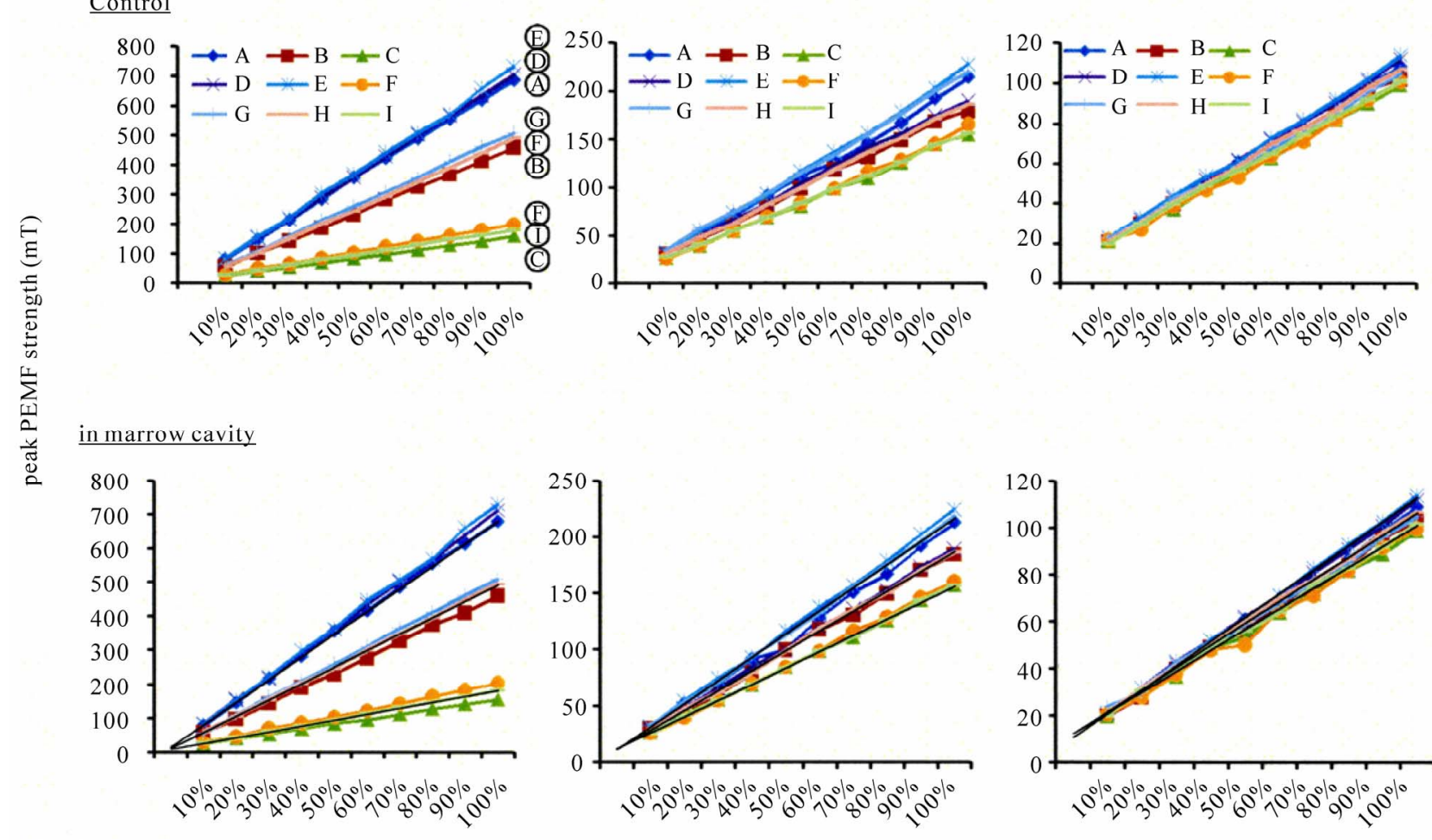

in marrow cavily
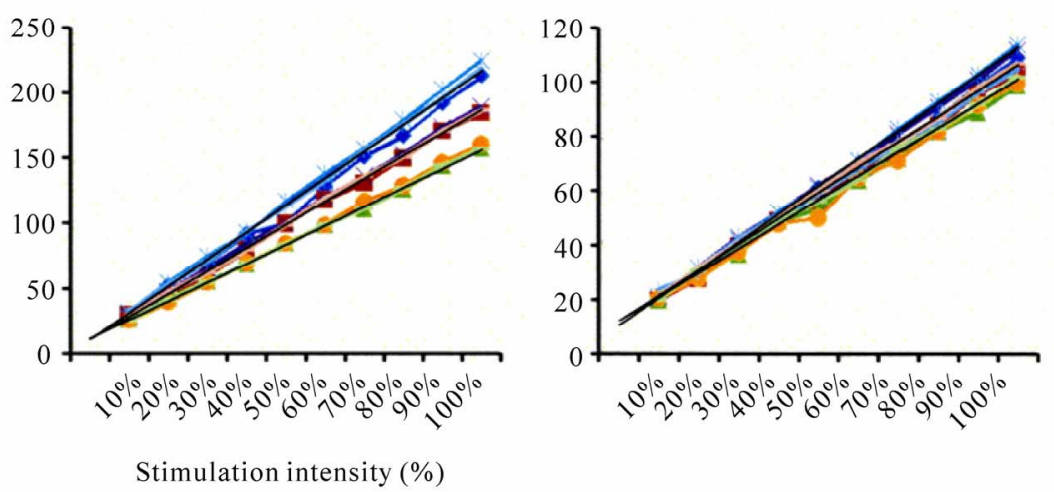

Figure 2. Relationships between peak PEMF strength and stimulation intensities in marrow cavity and control conditions. Note the 3 types of geometric difference in PEMF strength correlating with stimulation intensity and distance, with the highest values for positions A-D-E, middle values for B-G-H and lowest values for C-F-I. Positions A-I are the same as in Figure 1.

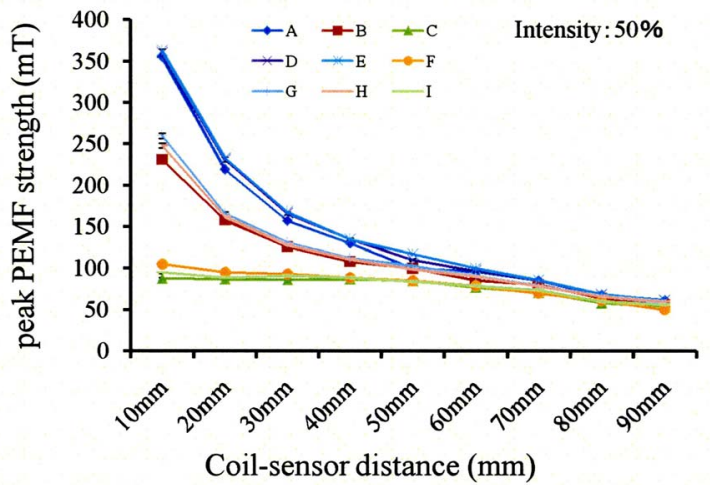

Figure 3. Relationships between peak PEMF strength and coilsensor distance at $50 \%$ stimulation intensity and at 9 positions (A-I).

were shown between each regression slope $(\mathrm{P}<0.0001)$ at a distance of 10-70 $\mathrm{mm}$. Values of regression slopes and those differences among groups decreased exponentially with increasing distance. The ratio of slope values in B-G-H and C-F-I groups to those in the A-D-E group were 0.72 and 0.26 at $10 \mathrm{~mm}$ distance, 0.86 and 0.71 at

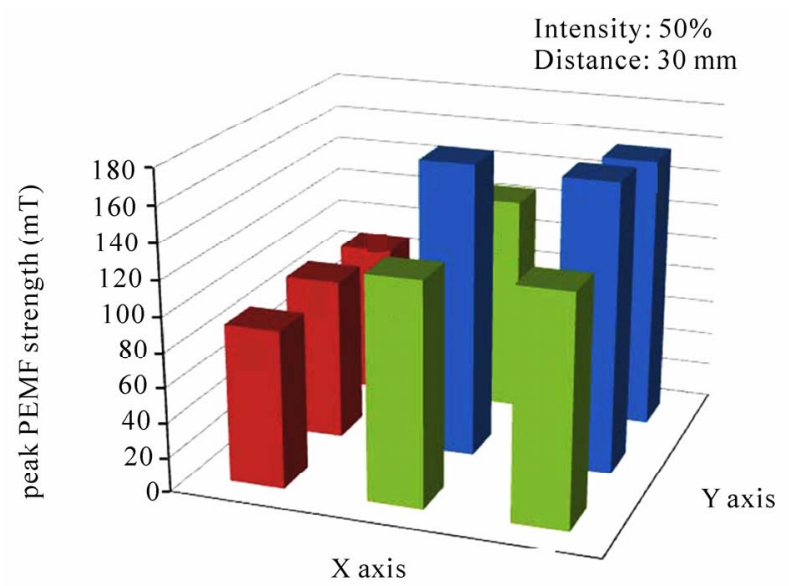

Figure 4. Relationships between peak PEMF strength and horizontal positions (A-I) at 50\% stimulation intensity and 30 $\mathrm{mm}$ coil-sensor distance.

$50 \mathrm{~mm}$ distance, and 0.93 and 0.88 at $90 \mathrm{~mm}$ distance, respectively. These ratios exceeded 0.8 at a distance of $>60 \mathrm{~mm}$. Comparison of the fit of non-linear curves revealed one curve for all data set in each position group. 
For control conditions, no significant differences in PEMF strength were observed under any conditions.

\section{DISCUSSION}

The present study demonstrated: 1) intensity dependency (i.e., PEMF strength correlated perfectly with stimulation intensity); 2) distance dependency (i.e., these characteristics diminished with increased exponentially coil-sensor distance); and 3) position dependency (i.e., PEMF strength distributions in bone were classified into 3 groups according to horizontal position).

The strong linear relationship seen between PEMF strength and stimulation intensity indicates stimulation intensity as a very accurate predictor of PEMF strength in marrow cavity with bone marrow in long bones. However, 3 types of geometric difference in regression line slopes and absolute values of PEMF strength were identified, with higher stimulation intensity obviously showing larger geometric differences in PEMF strength. For the condition of $50 \%$ stimulation intensity from a distance of $30 \mathrm{~mm}$, for example, our results indicate that PEMF strength was attenuated by approximately $32 \%$ and $45 \%$ at positions B-G-H and C-F-I compared to positions A-D-E, respectively. In addition, distance-de pendent declines in regression slopes and reductions in geometric differences suggest that a distance factor also influences the relationship between stimulation intensity and PEMF strength. The relationship between electric field strength and coil-cortex distance at 5-30 $\mathrm{mm}$ also reportedly shows exponential decay as a steep decrease with increasing stimulation distance [4]. In the present study, the relationships between PEMF strength and coil-sensor distance (10-90 $\mathrm{mm}$ range) showed exponential decay with a breaking point at a coil-sensor distance of around $30 \mathrm{~mm}$. This result suggests that the longer coil-sensor distance, the smaller the geometric difference in PEMF strength, particularly in terms of the uniformity of the stimulated area (i.e., a decrease in focality) at distances $>40 \mathrm{~mm}$. Testing the influence of PEMF stimulation on bone tissue in vivo $[6,7,11]$, stimulation conditions with lower spatial focality would be preferable to achieve approximately equal PEMF strength throughout the whole marrow cavity with bone marrow in long bones. To the best of our knowledge, this is the first study investigating PEMF strength based on the measurements in marrow cavity in long bones under 810 stimulation conditions. Our results might be helpful in determining stimulation programs and estimating the in vivo efficacy of PEMF in marrow cavity in long bones for research and clinical use. The characteristics of PEMF strength presented here also suggest the potential for in vivo application of our data to other tissues [12] by manipulating focality and stimulation intensity. Further studies are needed to clarify magnetic field strength properties in other tissues and animals.

\section{ACKNOWLEDGEMENTS}

This work was supported in part by a Grant-in-Aid for Scientific Research (C, project nos. 18200512 and 22500611) from the Japan Society for the Promotion of Science in 2010, and by a Grant-in-Aid for Developed Research (B, project No. H22B19) from the Niigata University of Health and Welfare in 2010.

\section{REFERENCES}

[1] Yamada, S., Guenther, H.L., and Fleisch, H. (1985) The effect of pulsed electromagnetic fields on bone cell metabolism and calvaria resorption in vitro, and on calcium metabolism in the live rat. International Orthopaedics, 9, 129-134.

[2] Sakai, Y., Patterson, T.E., Ibiwoye, M.O., Midura, R.J., Zborowski, M., Grabiner, M.D. and Wolfman, A. (2006) Exposure of mouse preosteoblasts to pulsed electromagnetic fields reduces the amount of mature, type I collagen in the extracellular matrix. Journal of Orthopaedic Research, 24, 242-253.

[3] Diniz, P., Shomura, K., Soejima, K. and Ito, G. (2002) Effects of pulsed electromagnetic field (PEMF) stimulation on bone tissue like formation are dependent on the maturation stages of the osteoblasts. Bioelectromagnetics 23, 398-405.

[4] Thielscher, A. and Kammer, T. (2004). Electric field properties of two commercial figure-8 coils in TMS: calculation of focality and efficiency. Clinical Neurophysiology, 115, 1697-708.

[5] Tamaki, H., Yotani, K., Yuki, A., Kirimoto, H., Sugawara, K., Jigami, H., Tsubaki, A., Onishi, H. and Ogita, F. (2010) Characterization of magnetic field strength in long bone during pulsed electromagnetic stimulation. Advances in Exercise and Sports Physiology, 15, 77.

[6] Takano-Yamamoto, T., Kawakami, M. and Sakuda, M. (1992) Effect of a pulsing electromagnetic field on demineralized bone-matrix-induced bone formation in a bony defect in the premaxilla of rats. Journal of Dental Research, 71, 1920-1925.

[7] Shen, W.W. and Zhao, J.H. (2010) Pulsed electromagnetic fields stimulation affects BMD and local factor production of rats with disuse osteoporosis. Bioelectromagnetics, 31, 113-119.

[8] Tamaki, H., Yotani, K., Yuki, A., Nishizawa, T., Tomori, K., Kirimoto, H., Onishi, H., Ogita, F. and Takekura, H. (2010) Effects of pulsed electromagnetic fields stimulation on gene expression related to bone formation in spontaneously hypertensive rats. 32nd Annual Meeting of American Society for Bone and Mineral Research, Toronto, 16, 56 .

[9] Lingwood, M.D., Siaw, T.A., Sailasuta, N., Ross, B.D., Bhattacharya, P. and Han, S. (2010) Continuous flow overhauser dynamic nuclear polarization of water in the fringe field of a clinical magnetic resonance imaging system for authentic image contrast. Journal of Magnetic Resonance, 205, 247-254. 
[10] Bland, J. M. and Altman, D.J. (1986) Regression analysis. Lancet, 1, 908-909.

[11] Marquez-Gamino, S., Sotelo, F., Sosa, M., Caudillo, C., Holguin, G., Ramos, M., Mesa, F., Bernal, J. and Cordova, T. (2008) Pulsed electromagnetic fields induced femoral metaphyseal bone thickness changes in the rat.
Bioelectromagnetics, 29, 406-409.

[12] Martino, C.F., Perea, H., Hopfner, U., Ferguson, V.L. and Wintermantel, E. (2010) Effects of weak static magnetic fields on endothelial cells. Bioelectromagnetics, 31, 296-301. 